\title{
A FINITELY-CONTAINING CONNECTED SET ${ }^{1}$
}

\section{P. M. SWINGLE}

In a previous paper an example has been given of a set which, for every integer $n \geqq 2$, is the sum of $n$ mutually exclusive connected subsets, but which is not the sum of infinitely many such subsets. ${ }^{2}$ Here it is proposed to give an example of a connected set which, for every integer $n \geqq 2$, is the sum of $n$ mutually exclusive biconnected subsets but which is not the sum of infinitely many mutually exclusive connected subsets. This example has the further property that, for every such $n$, it contains $n$ mutually exclusive connected subsets but it does not contain infinitely many such subsets, being thus a finitely-containing connected set. ${ }^{3}$ The method used will be a modification of that used by E. W. Miller to obtain a biconnected set without a dispersion point. ${ }^{4}$ The hypothesis of the continuum is assumed, and use is made of the axiom of Zermelo.

The method used by Miller is dependent primarily upon showing

1 Presented to the Society, April 15, 1939.

2 P. M. Swingle, Generalizations of biconnected sets, American Journal of Mathematics, vol. 53. (1931), pp. 387-388. I call such a set a finitely-divisible connected set. A connected set is defined here so as to contain at least two points. The example there given consists of a connected set which is the sum of infinitely many mutually exclusive biconnected subsets, each with a dispersion point, and a limit point of these subsets which none of them contains.

${ }^{3}$ Loc. cit., p. 395, Problem 7. This example also solves the questions raised in Problems 4, 5, and 6, pp. 394-395. Problem 2 was answered in part in American Journal of Mathematics, vol. 54 (1932), pp. 532-535. On p. 533 it is proved for $n=2$ that $E_{n}$ is the sum of $m$ mutually exclusive biconnected subsets where $m$ is an integer greater than $n$. And it is said that the proof is similar for $n>2$. For $E_{2}$ the proof depends upon constructing 3 biconnected sets, having only the origin in common. That a similar construction holds for any $E_{n},(n>1)$, is seen as follows. The half cones $x_{1}^{2}+x_{2}^{2}+\cdots+x_{n-1}^{2}=a x_{n}^{2},\left(x_{n} \geqq 0,-\infty<a<\infty\right)$, of $E_{n}$ are each $n-1$ dimensional surfaces. As each one is composed of concentric spheres $x_{1}{ }^{2}+x_{2}{ }^{2}+\cdots+x_{n-1}^{2}=r^{2}$ as is also $E_{n-1}$, each half cone and $E_{n-1}$ are topologically equivalent. As for $n=3, E_{n-1}$ is the sum of $n$ biconnected sets, with only the origin in common, a mathematical induction proof will show that this is true for $n>3$. For let the $a$ 's be divided into $C_{n+1, n}\left(C_{n+1, n}\right.$ is a binomial coefficient) mutually exclusive sets $N_{1}, \cdots, N_{c}$, each dense in their sum. Let, for each $a$ of $N_{i},(i=1, \cdots, c), x_{1}{ }^{2}+x_{2}{ }^{2}+\cdots+x_{n-1}{ }^{2}=a x_{n}{ }^{2}$ be the sum of parts of the same $n$ biconnected sets, where there is a total of $n+1$ such sets $B_{j}$, mutually exclusive except that they have the origin in common. Those $B_{j}$ 's determined by $N_{i}$ will be represented by the subscripts of that combination of $1,2, \cdots, n+1$, taken $n$ at a time, that $i$ of $N_{i}$ represents. Then the above is seen to be true.

${ }^{4}$ E. W. Miller, Concerning biconnected sets, Fundamenta Mathematicae, vol. 29, pp. 123-133. 
the existence of a widely connected subset $M$ of an indecomposable continuum $K$. It is only the part of this subset $M$ which is contained within a square $Q_{0}$ which causes $M$ to be biconnected and it is this fact which enables us to show the existence of the desired set of this paper. We will take a countable infinity of mutually exclusive such squares plus interiors, $Q_{1}, Q_{1}, Q_{2}, Q_{3}, \cdots$, each containing points of $K$ and having the relation with $K$ that Miller's square $A B C D$ has. We will use $Q_{i}$ as Miller does to show that a subset $B_{n i},(i=1,2, \cdots, n+1$; $n=1,2,3, \cdots)$, of a set $M$ is biconnected. And $Q$ will be used to show that there cannot be infinitely many mutually exclusive such subsets of $M$.

Let $V$ be a countable subset of $K$, which is dense in $K \cdot\left(Q_{1}+Q_{2}\right.$ $\left.+Q_{3}+\cdots+Q\right)$. Let $V_{i j},(i=1,2,3, \cdots ; j=1,2, \cdots, i+1)$, be a countable subset of $V$ everywhere dense in $V$ and such that (a) $V_{i j} \cdot V_{k l}$ is dense in $V$ if $i \neq k,(\mathrm{~b})$ for any $i$ the $V_{i k}$ 's, $(k=1,2, \cdots$, $i+1)$, are mutually exclusive, and (c) $V_{i 1}+V_{i 2}+\cdots+V_{i, i+1}=V$. For example $V_{11}$ and $V_{12}$ are mutually exclusive and $V_{11}+V_{12}=V$. Then $V_{11}$ is divided into three mutually exclusive subsets, each dense in $V$, one for each of the sets $V_{21}, V_{22}, V_{23}$ where $V_{2 j}$ is composed of such a set plus a similar subset of $V_{12}$. Each one of these three mutually exclusive subsets of $V_{11}$ is then divided into four mutually exclusive sets, each dense in $V$, to obtain the parts of $V_{31}, V_{32}, V_{33}, V_{34}$ contributed by $V_{11}$.

Let a division of $V$ into infinitely many mutually exclusive subsets be $U_{1}, U_{2}, \cdots$, where each $U_{t},(t=1,2, \cdots)$, is everywhere dense in $V$. Either (1) there exists a region $R$ of $Q$ and a $V_{i j}$ such that a $U_{t}$ contains $R \cdot V_{i j}$, or (2) there does not exist such an $R$. If (2) is true, $V_{i j}-U_{t} \cdot V_{i l}$ is dense in $V \cdot Q$ for each $i, j, t$. Consider case (1). Suppose for example that $U_{1}$ contains $R \cdot V_{32}$. Let $R_{1}$ be any region contained in $R$. Then $U_{1}$ contains a subset of $V_{r i},(r>3)$, which is dense in $V_{r j} \cdot R_{1}$, since $V_{32} \cdot R_{1}$ contains such a subset because of (a) above. Hence $U_{t},(t \neq 1)$, cannot contain a $V_{r j} \cdot R_{1}$, since $U_{1}$ and $U_{t}$ are mutually exclusive. Suppose now that there exist a $U_{t},(t \neq 1), U_{2}$ say, which contains a $V_{3 j} \cdot R_{1},\left(f \neq 2\right.$, but equals 1 say), for some $R_{1}$ of $R$. Hence as above $U_{t},(t \neq 2)$, does not contain a $V_{r i} \cdot R_{2}$, where $R_{2}$ is any region of $R_{1}$. There may exist now a $U_{t},(t \neq 1,2), U_{3}$ say, which contains a $V_{3 f} \cdot R_{2}$ for $f \neq 1,2$ but $f=3$ say. However since the $U_{t}$ 's are contained in $V_{31}+V_{32}+V_{33}+V_{34}$, there cannot exist a region $R_{3}$ of $R_{2}$ and a $U_{t},(t \neq 1,2,3)$, such that $U_{t}$ contains $R_{3} \cdot V_{3 f},(f \neq 1,2,3)$, for $R_{3} \cdot V_{34}$ must contain $R_{3} \cdot\left(U_{4}+U_{5}+\cdots\right)$. Thus in this case there exists an $R_{2}$ of $R$ such that there are at most three $U_{t}$ 's which contain a $V_{i j} \cdot R_{3}$, where $R_{3}$ is any region of $R_{2}$. Hence there exists an $R_{3}$ of $R$ 
and a $U_{t}, U^{\prime \prime}$ say, such that for every $V_{i j}, V_{i j}-V_{i j} \cdot U^{\prime \prime}$ is dense in $V \cdot R_{3}$. Therefore in both cases (1) and (2) above there exists a region $R^{\prime \prime}$ of $Q$ and a $U_{t}, U^{\prime \prime}$ say, such that for every $V_{i j}, V_{i j}-V_{i j} \cdot U^{\prime \prime}$ is dense in $V \cdot R^{\prime \prime}$.

The proof used by Miller to show that his widely connected set $M$ is biconnected is dependent upon having a countable subset $\Delta$ of $M$ and upon having a set of simple closed curves within the square $A B C D$ which have nothing in common with $M$ except points ${ }^{5}$ of $\Delta$. One of these simple closed curves is taken for each subset of $\Delta=V$ which is dense in $V \cdot R$, where $R$ is any region containing points of $V$. And the simple closed curve contains from the points of $V$ only points from this subset of $V \cdot R$. The set of such possible subsets is $c$, the power of the linear continuum.

Following the method of Miller arrange in a well ordered sequence the continua $C_{a}$ which separate $K$ :

$$
C_{1}, C_{2}, C_{3}, \cdots, C_{a}, \cdots, \quad a<\Omega_{c},
$$

where $\Omega_{c}$ is the first transfinite ordinal number to correspond to the cardinal number $c$ of the linear continuum. Let the regions of $Q$ be well ordered as well as the possible divisions $D_{1}, D_{2}, \cdots, D_{a}, \cdots$ of $V$ into infinitely many mutually exclusive subsets $U_{1}, U_{2}, \cdots$. As the power of this set of regions and the power of the set of $D_{a}$ 's are both $c$, let there be a one-to-one correspondence between each of these and the sequence $C_{1}, C_{2}, \cdots, C_{a}, \cdots$.

Choose for each $C_{a}$, having nothing in common with the interior of the square $Q$, a point set $M_{i a}$ for each $i$ and in each $Q_{i}$ construct a simple closed curve $J_{i a}$, exactly as Miller does for his $M$, using, for each $i, Q_{i} \cdot V$ in place of his ${ }^{6}(A B C D) \cdot \Delta$. Thus in $K$, exterior to $Q$, we have infinitely many mutually exclusive sets, $N_{1}, N_{2}, \cdots, N_{i}, \cdots$ say, each exactly similar to Miller's biconnected set $M$, except for $K \cdot Q$. In each region $R_{a}$ of $Q$ let a simple closed curve $J_{a}^{\prime}$ be constructed, by a method similar to that used by Miller, so that each $V_{i j}$ is dense in $K \cdot J_{a}^{\prime}$. Each infinite division $D_{a}$ above of $V$ determines a $U_{a}^{\prime \prime}$ and an $R_{a}^{\prime \prime}$ of $Q$ such that, for each $i, j, V_{i j}-V_{i j} \cdot U_{a}^{\prime \prime}$ is dense in $V \cdot R_{a}^{\prime \prime}$. In each $R_{a}^{\prime \prime}$ construct a simple closed curve $J_{a}^{\prime \prime}$ such that each $V_{i j}$ is dense in $K \cdot J_{a}^{\prime \prime}$ but $J_{a}^{\prime \prime} \cdot U_{a}^{\prime \prime}=0$. For each $C_{a}$ separating $Q \cdot K$ choose for each $V_{i j}$ a point or vacuous set, according to whether or not $C_{a} \cdot V_{i j}$ is vacuous, obtaining for each such $C_{a}$ an $M_{i j a}$ of $Q$ with the properties of Miller's $M_{a}^{\prime}$ 's. No $J_{a}^{\prime}+J_{a}^{\prime \prime}$ contains a point of an $M_{i j a}$ and no two $M_{i j a}$ 's consist of the same point.

${ }^{5}$ E. W. Miller, loc. cit., p. 129.

${ }^{6} \mathrm{E}$. W. Miller, loc. cit., pp. 128-130. 
The method used is dependent upon having chosen at any time during the process, under the hypothesis of the continuum, at most a countable infinity of points in $M \cdot\left(C_{1}+C_{2}+\cdots+C_{a}\right)$, where $M=N_{1}+N_{2}+\cdots+V+M_{111}+M_{121}+\cdots+M_{112}+M_{122}+\cdots$. This is true here just as it was for Miller's $M_{a}$ 's. As the set of composants of $K$ is of the power of the linear continuum, new points can always be chosen for new $C_{a}$ 's, and each choice can be made so that no composant contains more than one point of $M$.

The set $M$ is widely connected, for each $C_{a}$ contains at least one point of $M$ and no composant of $K$ contains more than one point ${ }^{7}$ of $M$. Let $B_{1 \rho},(g=1,2)$, contain all of $N_{o}+\left[V_{1 \rho}+\sum_{a=1}^{\Omega_{c}} M_{1 \rho a}\right] \times Q$, and let in addition $B_{11}$ contain all the rest of $M$, with the exception of the rest of $M$ in $Q_{1}$, and let $B_{12}$ contain this. Hence $B_{11}$ and $B_{12}$ are mutually exclusive sets whose sum is $M$. Each is connected, for every $C_{a}$ contains a point of each. Just as Miller showed, each $B_{1 g}$ is biconnected, for suppose that $B_{11}$, say, is the sum of the two mutually exclusive subsets $W_{1}$ and $W_{2}$. As $W_{1} \cdot V$ must be dense in $Q_{1} \cdot V$, there exists a $J_{1 a} \cdot M$ of $Q_{1}$ contained entirely in $W_{1} \cdot V$, according to the construction of the $J_{1 a}$ 's. As $B_{11}$ is widely connected, this is impossible. Hence $M$ is the sum of two mutually exclusive biconnected subsets $B_{11}$ and $B_{12}$.

In a similar manner for $n>1$ it is seen that $M$ is the sum of $n+1$ mutually exclusive biconnected subsets $B_{n 1}, B_{n 2}, \cdots, B_{n, n+1}$, where $B_{n j}$ contains $N_{j}+\left[V_{n j}+\sum_{a} M_{n j a}\right] \times Q$ of $M$ and $B_{n 1}$ contains all the rest of $M$, except the rest of $M$ contained in $Q_{1}$, and $B_{n 2}$ contains this.

It is seen however that $M$ is not the sum of infinitely many mutually exclusive connected subsets $T_{1}, T_{2}, \cdots$, for every region of $Q$ contains a $J_{a}^{\prime}$ and so each connected set $T_{i}$ would contain a $U_{i}$ dense in $V \cdot J_{a}^{\prime}$ and so dense in $V \cdot Q$. This $U_{i}$ is also dense in $V$ because of the $J_{i a}$ 's. Thus $T_{1} \cdot V, T_{2} \cdot V, \cdots$ is a division $D_{j}$ of $V$ into infinitely many mutually exclusive subsets $U_{1}, U_{2}, \cdots$ each dense in $V \cdot Q$. Hence one of these is a $U^{\prime \prime}$ which does not contain a point of some $J_{a}^{\prime \prime}$. Therefore the $T_{i}$, such that $U^{\prime \prime}=T_{i} \cdot V$, cannot be connected.

Thus it is seen that $M$ is an example of a finitely-divisible connected set and similarly of a finitely-containing connected set, since each connected subset of $M$ is widely connected.

New Mexico State College

${ }^{7}$ E. W. Miller, loc. cit., p. 126, Theorem 7. 